Revue internationale de l'économie sociale

Recma

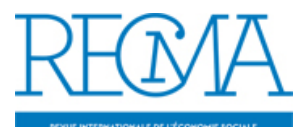

\title{
Rémunération en coopératives agricoles : les dessous du débat
}

\section{Maryline Filippi}

Numéro 341, juillet 2016

URI : https://id.erudit.org/iderudit/1037440ar

DOI : https://doi.org/10.7202/1037440ar

Aller au sommaire du numéro

Éditeur(s)

Association Recma

ISSN

1626-1682 (imprimé)

2261-2599 (numérique)

Découvrir la revue

Citer cet article

Filippi, M. (2016). Rémunération en coopératives agricoles : les dessous du débat. Revue internationale de l'économie sociale, (341), 119-125.

https://doi.org/10.7202/1037440ar d'utilisation que vous pouvez consulter en ligne.

https://apropos.erudit.org/fr/usagers/politique-dutilisation/ 


\title{
RÉMUNÉRATION EN COOPÉRATIVES AGRICOLES: LES DESSOUS DU DÉBAT
}

\author{
par Maryline Filippi*
}

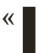

es coopératives payent moins que les sociétés de droit commer-

cial. " Ce reproche, adressé sur fond de crises des filières alimentaires françaises, questionne les raisons d'un prix payé par les coopératives à leurs associés coopérateurs moins rémunérateur que celui versé par les entreprises de droit commercial. Devrait-il toujours être supérieur et pourquoi? Dès lors, si cela n'était pas le cas, quel serait l'intérêt de maintenir ce modèle d'entreprise particulier, suspicieux en raison de sa propriété capitalistique, non soumis à l'impôt sur les sociétés? Ces interrogations soulèvent donc une question essentielle à l'essence du modèle coopératif. Examinons ce questionnement au regard des principes et du fonctionnement des coopératives agricoles. De quoi la rémunération des produits en coopérative est-elle le signe?

La propriété capitalistique d'une coopérative repose sur la détention par des associés coopérateurs de l'ensemble des parts de capital social de celle-ci en relation avec un engagement sur apport. Autrement dit: chaque associé détient du capital au prorata de l'activité réalisée par sa coopérative. Cette part de capital social lui donne droit à un "intérêt aux parts ", fixé statutairement à 1,80 \%. Cet intérêt aux parts est donc différent d'un intérêt d'une action, dont le taux varie en fonction des évolutions du marché boursier. La détention de capital donne également à l'associé un droit de vote qui, à la différence de celui octroyé par l'actionnariat, repose sur le principe "Une personne égale une voix ", distinct de celui " Une action égale une voix » (Hansmann, 1996). Ainsi, ce qui différencie fondamentalement la propriété capitalistique en coopérative de celle en droit commercial tient à: l'engagement sur activité obligatoire pour souscrire et détenir des parts de capital social; la rémunération fixe de la part sociale; l'expression démocratique où tous ont le même poids dans la prise de décision, quels que soient leur niveau d'engagement sur activité et le montant de capital détenu (Chomel et al., 2013).

Autrement dit, selon la définition du Code rural, les coopératives ont une propriété capitalistique dont les associés sont engagés sur activités, sont solidaires et décident collectivement de la stratégie productive, commerciale, mais également financière.

* Université de Bordeaux - Bordeaux Sciences Agro, UMR SAD-APT, université de Paris-Saclay, AgroParisTech. 
(1) La complexification des groupes coopératifs signifie un accroissement de leur taille (exprimée en termes de nombre de salariés et d'associés coopérateurs) mais fait surtout référence à la filialisation. Cette notion désigne le fait que, d'une part, les coopératives deviennent des groupes d'entreprises par leur détention de filiales de droit commercial qu'elles contrôlent seules ou à plusieurs et que, d'autre part, elles développent des structures partagées de type unions de coopératives, voire des holdings qui gèrent l'ensemble des participations financières qu'une coopérative détient dans d'autres entreprises. Cet ensemble compose le "périmètre coopératif ». Cette complexification des organisations a lieu également dans d'autres secteurs coopératifs, comme la banque ou le commerce.
Chaque année, lors de leur assemblée générale, et sur proposition du conseil d'administration, les associés coopérateurs votent ainsi la répartition des excédents de gestion de l'exercice annuel réalisé (i.e. du bénéfice), entre une distribution à tous au prorata des activités réalisées et une mise en réserve de tout ou partie au profit de la coopérative. Cette mise en réserve est essentielle, car elle vient en consolidation des fonds propres qui sont considérés comme structurellement faibles en coopérative (en raison de la faible contribution des membres). Elle revêt un caractère particulier: une fois ajoutée à ces fonds propres, cette mise en réserve ne pourra plus être repartagée entre tous, sauf en cas de liquidation ou de dévolution de la société. La mise en réserve abonde en effet les réserves dites impartageables, qui constituent une ressource « collective » à transmettre aux générations futures; en conséquence, elle ne sera pas intégrée au capital social récupéré par l'associé coopérateur lors de son départ de la coopérative. Cela explique l'expression de la coopérative comme outil transgénérationnel.

On comprend aisément qu'en temps de crise économique arbitrer pour céder une partie des excédents d'exploitation à la "coopérative ", au détriment de sa propre rémunération, puisse questionner les associés coopérateurs sur le bien-fondé et la nécessité d'un tel dessaisissement. Si la coopérative fait un bénéfice, pourquoi ne pas tout redistribuer aux associés coopérateurs? En outre, au fil du temps, sous l'effet de la complexification des organisations coopératives ${ }^{(1)}$, l'adhésion au projet collectif constitutif de la coopérative s'est forcément souvent amoindrie. De même, l'hétérogénéité entre les membres, en particulier dans les coopératives polyvalentes, a crû, renforçant la difficulté de décider collectivement de l'affectation des résultats. C'est ce que la littérature économique désigne sous le terme du « dilemme coopératif » relatif à la question des droits de propriété et à l'exercice de la gouvernance coopérative (à titre d'exemple, Hansmann, 1996; Hendrikse, Bijman, 2002; Chaddad, Cook, 2004).

\section{L'ajustement au « prix du marché »}

La politique pratiquée en matière de rémunération des produits est pourtant perçue comme un indicateur de la "gouvernance coopérative ».

A la création des coopératives, l'idée de se regrouper visait la recherche d'une meilleure "rémunération des produits " (production ou service), selon un modèle de coopérative assimilé à celui des coopératives de type collecte-approvisionnement. L'augmentation des volumes collectés était réalisée dans l'objectif économique d'accroître les économies d'échelle, afin de diminuer les coûts de collecte ou d'améliorer le pouvoir 
(2) La loi d'avenir pour l'agriculture, l'alimentation et la forêt de 2014 comprend une série de modifications concernant la coopération agricole, afin d'en améliorer le fonctionnement et la gouvernance, et modifie ainsi les articles L.521-1 et suivants du Code rural. Rappelons qu’en matière de rémunération «l'organe chargédel'administration de la société (i.e. le conseil d'administration) définit les modalités de détermination et de paiement du prix des apports de produits, des services ou des cessions d'approvisionnement, notamment les acomptes et, s'il y a lieu, les compléments de prix, et propose une répartition des excédents annuels disponibles. Cette répartition est décidée par l'assemblée générale ordinaire» (loi n 47-1775 du 10 septembre 1947).

(3) Terre-net.fr/actualite-agricole/ politique-syndicalisme/article/ la-gouvernance-des-cooperativesreprise-en-main-205-93192.htm, Terre-Net, 17 novembre 2014 de négociation en termes de prix auprès des négociants et autres transformateurs situés à l'aval des filières. Aussi, la distribution totale des excédents était une pratique courante, considérée comme habituelle. L'exonération de l'impôt sur les sociétés s'avère être la contrepartie fiscale, en dédommagement de l'obligation pour la coopérative de collecter l'intégralité des produits des associés, et cela quelles que soient les conditions de coûts, y compris lorsque cette collecte est réalisée à perte. Cette obligation introduit une différence significative avec les entreprises de droit commercial, qui, non tenues par cette obligation, rentabilisent économiquement et de façon rationnelle leur activité.

Dans cette perspective, le "revenu d'un associé coopérateur » provient du paiement des produits apportés à la coopérative tenue de tout collecter (selon la règle réciproque de l'apport total). Le prix payé au producteur se compose du prix du produit apporté (versé sous forme d'acompte) et d'un complément de prix ajustant, en fin de période, le prix réellement obtenu lors de la cession par la coopérative de la production ${ }^{(2)}$. Le revenu est ensuite complété par la rémunération des parts sociales. Dans le cas des groupes coopératifs, s'il y a lieu, une distribution du dividende est possible en provenance d'une filiale bénéficiaire et dont le conseil d'administration de la coopérative aura décidé de faire remonter aux associés coopérateurs tout ou partie des bénéfices. La loi de modernisation de 2014 ajoute au Code rural un nouvel article (L521-3-1) portant sur les modalités de détermination et de paiement du prix des apports, des services ou de cessions d'approvisionnement par l'assemblée générale de la coopérative sur proposition du conseil d'administration (ou du directoire s'il y a lieu), i.e. des prix d'achat, des acomptes et la prise en compte des variations des fluctuations des prix des matières premières, constatées à partir d'indicateurs publics. Ainsi, les coopératives devront déterminer "des critères relatifs aux fluctuations des prix des matières premières agricoles et alimentaires influençant le prix de production de ces produits qui les conduiront à délibérer sur la prise en compte de cesfluctuations dans le calcul du prix de collecte de ces produits ${ }^{(3)} »$. Cette « clause miroir ", en permettant la renégociation du prix des apports, introduit donc la possibilité de partager les charges liées à la volatilité du prix des matières premières. Elle participe à une meilleure transparence des relations entre la coopérative et ses associés, dont celle qui permet d'améliorer l'information auprès de ces derniers.

Initialement, la politique des coopératives a effectivement été conduite dans l'optique de rémunérer au mieux les produits des associés coopérateurs. Pourtant, l'exigence de rentabilité pour les coopératives a progressivement conduit les administrateurs à proposer une diminution de la rémunération du produit 
(4) Dans d'autres pays européens comme en Allemagne ou aux PaysBas, les coopératives sont autorisées à avoir dans leur capital social des investisseurs financiers. Les EtatsUnis disposent de sept statuts différents. C'est l'une des raisons qui justifient l'appellation de « modèle traditionnel ». Les filiales étant de droit commercial, la composition des détenteurs du capital social peut panacher des banques, des industriels, des fonds de pension... pour l'ajuster au "prix du marché ». La raison principale tient au fait qu'avec des fonds propres faibles, rendant les banques moins enclines à accorder des crédits, le financement du fonctionnement des outils de transformation et de distribution s'est appuyé sur l'activité même des associés coopérateurs, via leur mise en réserve (Filippi et al., 2006). Par ailleurs, la pratique de l'alignement sur le " prix du marché » permet un pilotage plus rigoureux de la coopérative et de sa filiale lorsque cette dernière achète la production des associés coopérateurs collectée par la coopérative (voir le système de prix de cession en interne). Cela est motivé de plus en plus par la possibilité ainsi offerte de faire entrer au capital de la filiale de droit commercial des investisseurs extérieurs, industriels ou financiers (Mauget, 2008). En France, en effet, le statut considéré comme « traditionnel » des coopératives conduit à n'avoir que des associés coopérateurs engagés sur activité au capital de la coopérative, ce qui ne l'est pas dans leurs filiales de droit commercial (Filippi, Cook, 2014) ${ }^{(4)}$.

La répartition des bénéfices des filiales, une fois la part de bénéfice remontée à la coopérative, se réalise en fonction du choix des associés coopérateurs de la "clé de répartition" (affectation en réserve et distribution en fonction des apports). La politique pratiquée en matière de rémunération des produits a ainsi dû prendre en compte les " charges de structure » de la coopérative qui, en s'accroissant, viennent grever l'excédent susceptible d'être distribué aux associés coopérateurs. Dès lors, le développement des filiales, motivé par l'investissement dans les activités de transformation et de distribution à l'aval des filières et par la recherche de création et de captation de valeur ajoutée, nécessite l'introduction de nouveaux métiers et exige de nouvelles compétences (en finance, management, marketing, recherche-développement, etc.). Cette évolution des coopératives génère, en contrepartie, des coûts de structure par rapport au modèle initial de base des coopératives de type collecte-approvisionnement. Cette question rejoint celle de l'organisation efficiente de la coopérative sous forme de groupe et de ses niveaux, coopérative et filiale. Elle traduit aussi le glissement économique de la seule activité de collecteapprovisionnement vers la prise en considération des activités de transformation.

\section{Rémunération et autres critères de performance}

En conséquence, la revendication pour un prix plus rémunérateur se confronte à l'équilibre entre structure coopérative et création de valeur. Le débat a tourné dans l'arène médiatique autour de la " distanciation » entre la coopérative 
et ses membres. La question porte finalement tout autant sur le fonctionnement d'une coopérative que sur sa capacité à capter une part plus équitable de la valeur créée au sein des filières alimentaires. Prendre en compte la revendication juste des associés coopérateurs pour une meilleure rémunération questionne finalement la capacité d'une coopérative à concilier rémunération de ses associés et compétitivité économique, opérée dans le cadre des marchés mondialisés et des (dé)régulations publiques accompagnant les évolutions de l'agriculture et de l'agroalimentaire.

Le mode d'affectation des résultats étant clarifié, revenons sur les droits et devoirs des associés de la coopérative vis-à-vis des associés coopérateurs, et vice versa. Le droit des associés est de recevoir des bénéfices individuels et collectifs. La remise en cause du modèle coopératif sous l'angle d'une rémunération insuffisante des associés ne semble cependant pas être posée de façon pertinente. Au-delà des erreurs possibles inhérentes à toute entreprise (y compris coopérative) en termes de gestion d'entreprise ou de stratégie de positionnement marché, la non-réalisation des objectifs économiques est aussi liée au prix obtenu dans un contexte de compétition mondialisée exacerbée. Choisir un positionnement marché de produits standardisés, plutôt que de produits de qualité particulière, expose inévitablement la stratégie productive aux règles de compétitivité associées.

Le prix en coopérative n'est toutefois pas le seul critère qui permet d'évaluer le bien-fondé du modèle (Jarrige, Touzard, 2001). Comme le rappelle la Food and Agriculture Organization (FAO) des Nations unies, la finalité, autrement dit la raison sociale de la coopérative (être au service de l'associé coopérateur), intègre d'autres "dimensions de performance ", comme l'accès au marché, la sécurité alimentaire et la différenciation par la qualité des produits. La coopérative se doit d'être performante économiquement et socialement pour l'ensemble de ses associés coopérateurs et pour la société.

Cela génère cependant des "devoirs réciproques » des associés envers leur coopérative, comme réaliser les apports annoncés alors que la volatilité des prix offre des rémunérations plus intéressantes en livrant à des négoces ou s'impliquer plutôt que déléguer passivement la bonne gestion de la coopérative à un nombre restreint d'administrateurs. Poser la question du montant de la rémunération oriente le débat vers les modalités de gouvernance des coopératives, des indicateurs spécifiques de la performance coopérative et vers les choix en matière de stratégie d'entreprise (Filippi et al., 2009; Bijman et al., 2014).

La question de la solidarité coopérative s'exerce dans les deux sens, aussi est-il essentiel de remettre en discussion au cœur des entreprises leur « projet coopératif ». Les associés sont-ils 
en réelle capacité de connaître, de comprendre et de décider de la stratégie de leur coopérative? Les coopératives sont-elles suffisamment à l'écoute et en capacité de concilier les intérêts divergents, voire potentiellement contradictoires, de leurs associés coopérateurs?

Ainsi, cette discussion autour du "projet coopératif » devrait également tenir compte des autres acteurs des chaînes de valeur, y compris de la distribution et des consommateurs, afin de construire des dispositifs à l'articulation des filières et des territoires, non seulement pour faire face à l'hyperconcurrence et parfois à une dérégulation destructrice des marchés, mais surtout pour relever les challenges actuels du secteur agricole et agroalimentaire.

En posant la question de la création de valeur, de la gouvernance coopérative et de la tension entre compétitivité économique et dimension sociale, cette rémunération en coopérative agricole interpelle au final aussi les pratiques des autres familles d'activité de l'économie sociale et solidaire. 


\section{BIBLIOGRAPHIE}

Bijman J., Hanisch M., Van der Sangen G., 2014, " Shifting control? The changes of internal governance in agricultural cooperatives in the EU ", Annals of Public and Cooperative Economics, 85 (4), p. 641 - 661.

Chaddad F. R., Cook M. L., 2004, « Understanding new cooperative models: An ownership control rights typology ", Review of Agricultural Economics, 26 (3), p. 348-360.

Chomel C., Decleck F., Filippi M. et al., 2013, Les coopératives agricoles, identité, gouvernance et stratégies, Bruxelles, Larcier, $491 \mathrm{p}$.

Filippi M., Cook M.L., 2014, " How does Public Policy support explain agricultural cooperatives'development? Comparison between France and US ", in Cooperatives'Power of Innovation, Sommet international des coopératives, Québec, p. 727-747.

Filippi M., Frey O., Mauget R., 2009, «Les coopératives agricoles face à l'internationalisation et la mondialisation des marchés ", Recma, n³10, p. 31-51.
Filippi M., Frey O., Triboulet P. et al., 2006, "Bilan des lois de 1991 et 1992 et gouvernance des groupes coopératifs ", rapport final, ministère de l'Agriculture et de la Pêche, 105 p.

Hansmann H., 1996, The Ownership of Enterprise, Harvard University Press.

Hendrikse G., Bijman J., 2002, « Ownership structure in agrifood chains: The marketing cooperative ", American Journal of Agricultural Economics, 84 (1), p. 104 - 119.

Jarrige F., Touzard J.-M., 2001, « Les mutations de l'organisation coopérative à travers l'évolution de ses règles, la rémunération de la vendange dans les caves coopératives du Midi ", Recma, n²80, p. 36-48.

Mauget R., 2008, « Les coopératives agricoles, un atout pour la pérennité de l'agriculture et dans la mondialisation ", Recma, $n^{\circ} 307$, p. 46-57. 